\title{
A Mathematical Model of the Strain and Stress Kinetics during Welding of Thin-Walled Products
}

\author{
Gocha Gubeladze ${ }^{1}$, Paata Geradze ${ }^{2}$ \\ ${ }^{1}$ Kutaisi Technological Academy, 4600 Kutaisi, Georgia \\ ${ }^{2}$ Akaki Tsereteli State University, Engineering-Technical faculty, 4600 Kutaisi, Georgia
}

\begin{abstract}
The paper dwells on the mathematical model of the strain and stress of the elements of the thin-walled systems. A version of the sophisticated theory of shells with the use of several base surfaces has been developed at the Kutaisi Technical University [3,8]. The theory is based on a kinematic hypothesis thereby facilitating the construction of a three-dimensional field of deformation of shell by deformation of two or more surfaces. The use of several base surfaces allows not only for accounting the transverse shears and crimping, but also, with account for the shell thickness, for modeling the mechanical and thermal phenomena on the front surfaces of the layers. In doing so, the geometrical and mechanistic interpretation of generalized displacements and generalized internal forces is clear enough, and the basic equations are simple. The model is based on a geometrically linear version of the theory of shells with the use of several base surfaces and the theory of non-isothermal plastic flow [4]. The developed mathematical model of the strain and stress kinetics allows for evaluating the temperature and strain-stress states of thin-walled products during welding.
\end{abstract}

\section{Introduction}

Theoretical studies of welding deformations and stresses give rise to considerable difficulties due to a mixed picture of the interrelated physicomechanical and chemical phenomena occurring in the material during welding $[1,2]$. It is necessary to mention that the welding production is distinguished by the variety of both the products themselves (materials, geometrical dimensions, shapes, etc.) and external effects (methods for the supply and capacity of energy being supplied, methods of spatial registration of products, etc.). In such conditions, one cannot use the simplified approaches because of inadequacies of the appropriate mathematical model.

The mechanical properties of weldable thin-walled structures are studied theoretically by a theory of the elastoplastic plates and shells. However, an analysis of the published works shows that the elastoplastic shells are little-investigated from the perspectives of the sophisticated theories, while the results of a number of works highlight the importance of including the transverse shears and crimping in plasticity problems. A version of the sophisticated theory of shells with the use of several base surfaces has been developed at the Kutaisi Technical University [3]. The theory is based on a kinematic hypothesis thereby facilitating the construction of a three-dimensional field of deformation of shell by deformation of two or more surfaces. The use of several base surfaces allows not only for accounting the transverse shears and crimping, but also, with account for the shell thickness, for modeling the mechanical and thermal phenomena on the front surfaces of the layers. In doing so, the geometrical and mechanistic interpretation of generalized displacements and generalized internal forces is clear enough, and the basic equations are simple.

\section{Methodology}

The model is based on a geometrically linear version of the theory of shells with the use of several base surfaces and the theory of non-isothermal plastic flow [4]. The geometrical linearity denotes similarity of the metrics of the deformed and undeformed states of shell, and the possibility of using the linear tensor of deformations [5]:

$$
\varepsilon_{i j}=\frac{1}{2}\left(\nabla_{i} U_{j}+\nabla_{j} U_{i}\right)
$$

where $U_{-} \mathrm{j}$ - the components of the displacement vector; $\nabla \_j-$ operator of covariant differentiation.

Since the kinematic hypothesis uses not one, but two base surfaces, there have been introduced specific parameters of the shell space associated with both surfaces (Fig. 1):

$$
\vec{R}\left(X^{1}, X^{2}, X^{3}\right)=\stackrel{(-)}{\vec{R}}\left(X^{1}, X^{2}\right)+X^{3}\left(\stackrel{(+)}{\vec{R}}\left(X^{1}, X^{2}\right)-\stackrel{(-)}{\vec{R}}\left(X^{1}, X^{2}\right)\right) .
$$




$$
\stackrel{(+)}{\vec{R}}\left(X^{1}, X^{2}\right) \text { and } \stackrel{(-)}{\vec{R}}\left(X^{1}, X^{2}\right)
$$

set the parameters of the shell space base surfaces. Let us $(-)$ call conditionally the first surface $\stackrel{(-)}{S}$ the lower base surface, but the second one $\stackrel{(-)}{S}$ - the upper surface. The vector

$$
\vec{H}\left(X^{1}, X^{2}\right)=\stackrel{(+)}{\vec{R}}\left(X^{1}, X^{2}\right)-\stackrel{(-)}{\vec{R}}\left(X^{1}, X^{2}\right)
$$

Which is perpendicular to the surface $S$, assigns the points of the base surfaces with the same Gaussian coordinates $\left(X^{1}, X^{2}\right)$.

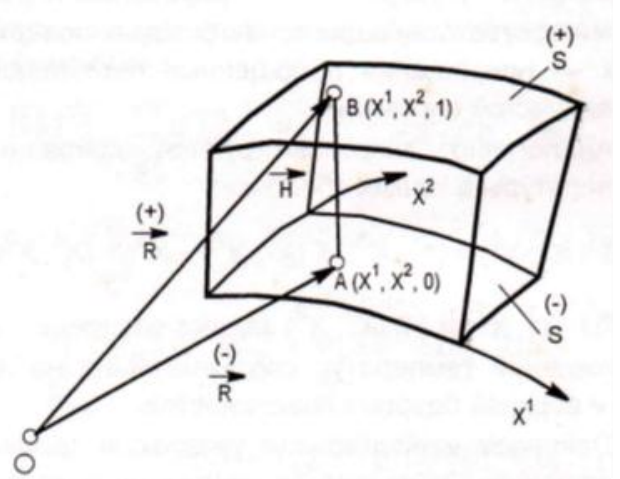

Figure 1. The parameters of the shell space with two base surfaces.

There have been accepted the following designations: the Latin indexes 1, 2, 3, but the Greek ones - 1, 2; the magnitudes with a negative sign (-) are set on the lower base surface, and the magnitudes with a positive sign $(+)$ - on the upper base surface; the index with a negative sign (-) means that tensor component by this index is referred to a basis vector on the lower base surface, and with a positive sign $(+)$ - on the upper base surface.

The main ratios of the theory of non-isothermal plastic flow, linking the increments of the components of deformation tensor $\delta \sigma^{i j}$ with the increments of the components of deformation tensor $\delta \varepsilon_{k m}$ can be written as the defining relationships for an anisotropic elastic body [4]:

$$
\delta \sigma^{i j}=A^{i j k m}\left(\delta \varepsilon_{k m}-\alpha_{k m} \delta T\right),
$$

where, $A^{i j k m}$ - the components of tensor of the

elastoplastic properties depending on the stress state, temperature and the achieved degree of hardening in a given point of the shell; $\alpha_{k m}$ - the components of tensor

of the temperature linear expansion coefficients.

The formula (5) necessitates representation of the equations of the theory of shells with the use of several base surfaces in the increments as well. According to the main kinematic hypothesis, the points of the shell receive the additional displacements:

$$
\delta \vec{U}\left(X^{1}, X^{2}, X^{3}\right)=\left(1-X^{3}\right) \delta \stackrel{(-)}{\vec{U}}\left(X^{1}, X^{2}\right)+X^{3} \delta \vec{U}\left(X^{1}, X^{2}\right),
$$$$
(-) \quad(+)
$$

where $\vec{U}\left(X^{1}, X^{2}\right), \delta \vec{U}\left(X^{1}, X^{2}\right)$ - the increments of the displacements of the appropriate pints of the base surfaces-the increments of the generalized displacements of the mechanical systems.

The temperature increments in the shell points are approximated similarly:

$$
\delta T\left(X^{1}, X_{(-)}^{2}, X^{3}\right)=\left(\begin{array}{c}
\left.1-X^{3}\right) \\
\delta
\end{array} \stackrel{(-)}{T}\left(X^{1}, X^{2}\right)+X^{3} \delta \stackrel{(+)}{T}\left(X^{1}, X^{2}\right)\right.
$$

where $\delta T\left(X^{1}, X^{2}\right), \delta T\left(X^{1}, X^{2}\right)$ set the distributions of the temperature increments on the lower and upper base surfaces, accordingly.

The basic variational equation of the theory in the increments derived from the principle of possible displacements, has the form as follows:

$$
\begin{aligned}
& \int_{(-)}\left[\left(\delta \stackrel{(-)}{t^{\bar{\beta}} \bar{\imath}} N_{\beta}-\delta{ }^{(-)} \bar{\imath}\right) \delta U^{(-)}\right. \\
& \left.+\left(\delta t^{\Gamma}{ }^{\bar{\beta}} \stackrel{(-)}{N_{\beta}}-\delta \stackrel{(+)}{T} \bar{\imath}\right) \delta \stackrel{(+)}{U}_{\bar{\imath}}\right] d \stackrel{(-)}{\Gamma} \\
& -\int_{\substack{(-) \\
S}} \delta U_{i}^{(-)}\left[\left(\nabla_{\beta} \delta t^{(-)} \bar{\beta} \bar{i}+\delta \vartheta^{\bar{i}}+\delta \stackrel{(-)}{\kappa^{i}}\right)\right.
\end{aligned}
$$

$\left.+\delta U_{i}^{(+)}\left(\nabla_{\beta} \delta \stackrel{(+)}{t} \bar{\beta} \bar{i}^{-} \delta \vartheta^{\bar{i}}+\delta \stackrel{(+)}{\kappa}^{i}\right)\right] d \stackrel{(-)}{S}=0$,

where ${ }^{(-)} \bar{\beta} \bar{\imath}, \stackrel{(+)}{t} \bar{\beta} \bar{\imath}-$ the increments of the generalized internal forces, corresponding with the increments of stresses acting on the transverse shell areas; $N_{-}^{(-)}$the $(+)$ outer normal unit vector to a border $\Gamma$ placed on the tangential plane to a surface $\stackrel{(-)}{S}$ and equal to ${ }_{N} \vec{R}^{\beta}$; $(+) \quad(+)$ $\delta \mathrm{T}, \delta \mathrm{T}-$ the increments of the generalized forces, conditioned by the increments of the external surface $(-)$ forces acting on the transverse boundary surfaces; $\Gamma-$ the border of the internal base surface; $\delta \vartheta^{\bar{m}}-$ the increments of the generalized internal forces, corresponding with the increments of the external volumetric and external surface forces applied to the appropriate surfaces.

It follows from the equation (8) that, by reason of $(+) \quad(-)$ the arbitrariness of $\delta U_{\bar{l}}$ and $\delta U_{\bar{\iota}}$ for the internal points $(-)$ of the base surface $S$ :

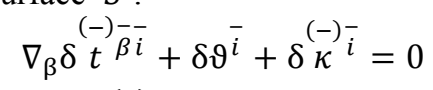

$\nabla_{\beta} \delta^{(+)}{ }^{\bar{\beta}} \bar{i}-\delta \vartheta^{\bar{i}}+\delta \stackrel{(+)}{\kappa} \bar{i}=0$

Based on the formulas (1) and (5), for the increments of the internal generalized forces, we can obtain the decompositions:

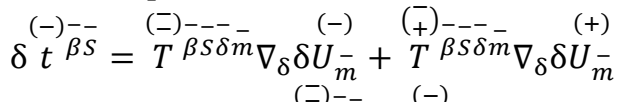

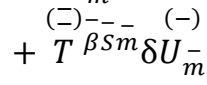

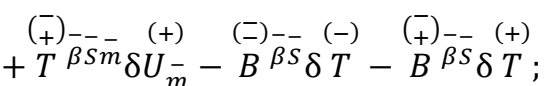




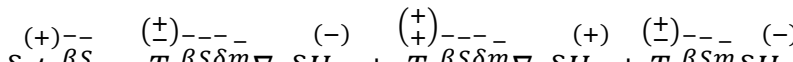

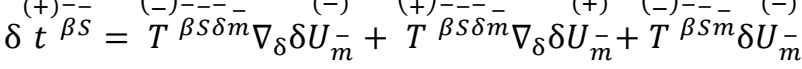

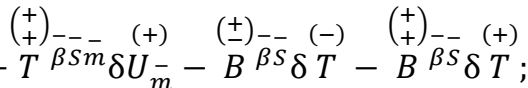

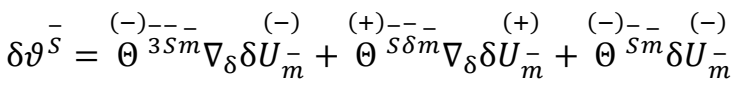

$$
\begin{aligned}
& +\stackrel{(+)}{\Theta}^{S m} \delta U_{-}^{(+)}-\stackrel{(-)}{\Xi} \bar{S}^{(-)} \delta T-\stackrel{(+)}{\Xi}^{S} \delta{ }^{(+)},
\end{aligned}
$$

whose coefficients :

$$
\begin{aligned}
& T^{(\bar{z}) \bar{S} \delta \bar{m}}=\int_{0}^{1} \frac{A^{\bar{i} \bar{j} \delta \bar{m}}+A^{\bar{i} \bar{j} \bar{m} \bar{\delta}}}{2} \cup\left[\left(1-\mathrm{X}^{3}\right) \mathrm{G}_{i}-\mathrm{G}_{j}^{\alpha}\right. \\
& \left.\mathrm{X}^{3} \mathrm{G}_{i}^{\beta} \underset{j}{\beta} \mathrm{G}_{j}^{\underline{\gamma}} \mathrm{G}_{+}^{-\bar{\alpha}}\right]\left(1-\mathrm{X}^{3}\right)^{2} \mathrm{dX} \mathrm{X}^{3}, \\
& \stackrel{( \pm ⿱ 亠 䒑}{T}^{\bar{\beta} \overline{3} \bar{m}}=\int_{0}^{1} \frac{A^{\bar{i}-\bar{j} \bar{n}}+A^{\bar{i} \bar{j} \bar{n}}}{2} \cup\left[-\delta_{\bar{n}}^{\bar{m}}\right. \\
& \left.-\left(1-X^{3}\right) \Gamma_{n 3}^{\mathrm{m}}\right]\left[\mathrm{G}_{i}^{0} \mathrm{G}_{j}^{-}{ }_{j}^{3}+\mathrm{X}^{3} \mathrm{G}_{i}^{\beta} \mathrm{G}_{j}^{\frac{\gamma}{\gamma}} \mathrm{G}_{\gamma}^{-\overline{3}}\right] \mathrm{X}^{3} \mathrm{~d} \mathrm{X}^{3} \text {, }
\end{aligned}
$$

$$
\begin{aligned}
& \stackrel{(+)-\bar{\Theta}}{\Theta^{3 \delta}}=\int_{0}^{1} \frac{A^{\bar{i} \bar{j} \delta \bar{m}}+A^{\bar{i} \bar{j} \bar{m} \delta}}{2} \cup\left[\mathrm{G}_{i}^{3} \mathrm{G}_{j}^{3}\right. \\
& \left.+\mathrm{X}^{3} \mathrm{G} \underset{i}{\stackrel{3}{-}} \underset{j}{-} \mathrm{G}_{\gamma}^{\gamma} \underset{\gamma}{\overline{3}}\right] \mathrm{X}^{3} \mathrm{dX} \mathrm{X}^{3}, \\
& \stackrel{( \pm)}{\Xi} \bar{\alpha}=\int_{0}^{1} A^{\bar{i} \bar{j} k \bar{m}} \alpha_{k \bar{m}}^{-\bar{v}} \cup\left[\left(1-\mathrm{X}^{3}\right) \mathrm{G}_{i}^{3} \mathrm{G}_{j}^{\alpha}\right. \\
& \left.+\mathrm{X}^{3} \mathrm{G}_{i}^{3} \underset{j}{\stackrel{3}{-}} \underset{\gamma}{\underline{\gamma}} \mathrm{G}_{+}^{\bar{\alpha}}\right] \mathrm{X}^{3} \mathrm{dX} \mathrm{X}^{3},
\end{aligned}
$$

where,

$$
U=\sqrt{\frac{G}{(-)} G--}
$$

depend on the shell geometry, the adopted parameters of its space, thermo-mechanical characteristics of the material and, most importantly, the achieved stress state, temperature and the accumulated strain measure in the shell points $\left(G_{i j}-\right.$ the components of metric tensor, $\Gamma_{i j}^{k-}$ the Christoffel symbols).

Applying the formulas (10) to the equation (8), we obtain the system of differential equations, linear with respect to the increments of displacements $\delta \stackrel{(-)}{U}_{\bar{\imath}}$ and $\delta \stackrel{(+)}{U}_{\bar{\imath}}$ - of the points of the base surfaces:

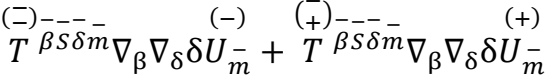

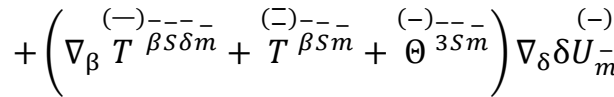

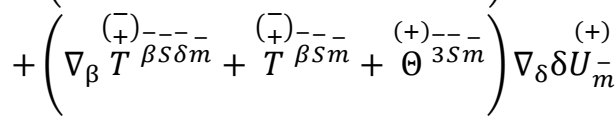

$$
\begin{aligned}
& +\left(\nabla_{\beta} \stackrel{(-)}{T} \bar{\beta} \overline{S m}^{-\stackrel{(-)}{\Theta} \overline{S m}}\right) \nabla_{\delta} \delta U_{\bar{m}}^{(-)}
\end{aligned}
$$

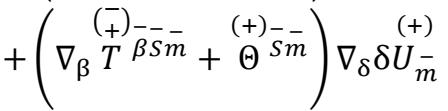

$$
\begin{aligned}
& +\delta \stackrel{(-)}{\kappa}^{S}-\left(\nabla_{\beta} \stackrel{(-)}{B}^{\beta} \bar{S}+\stackrel{(-)}{\Xi} \bar{S}\right) \delta \stackrel{(-)}{T}-\stackrel{(-)}{B} \bar{B} \bar{S} \frac{\partial \delta{ }^{(-)}}{\partial \mathrm{X}^{\beta}}
\end{aligned}
$$

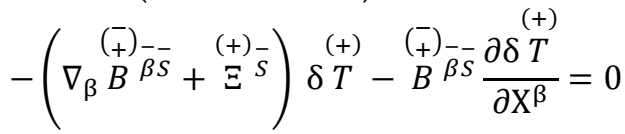

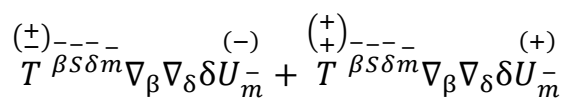

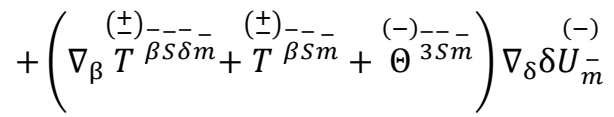

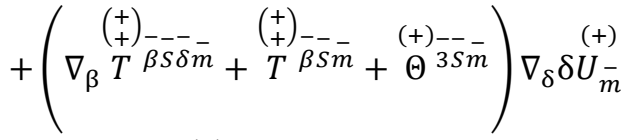

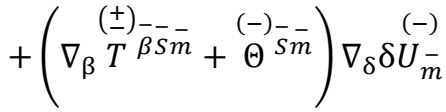

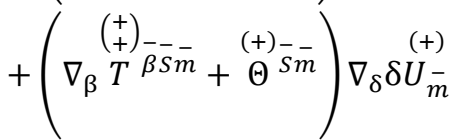

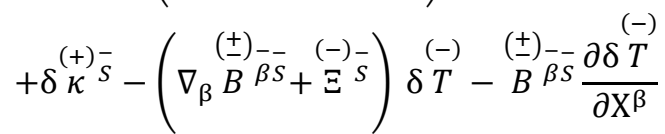

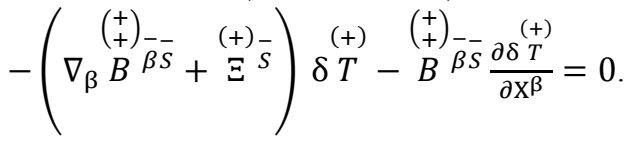

The extracted equations are complex, and the only way to solve them is to develop the numerical algorithms, particularly on the basis of the finite elements method [6].

Let us decompose the domain of integration $S$ into the finite elements and consider one finite element $\stackrel{(-)}{S}(e)$. Let us use the displacement fields of the points of the base surfaces on the element [6]:

$$
\delta \stackrel{(-)}{U_{-}^{-}}=\delta \stackrel{(-)}{U} \stackrel{N}{i}_{i} \Psi_{N}\left(\xi^{1}, \xi^{2}\right) ; \quad \stackrel{(+)}{\delta U_{-}^{-}}=\delta \stackrel{(+)}{U} \frac{N}{i} \Psi_{N}\left(\xi^{1}, \xi^{2}\right),
$$

where $\Psi_{N}\left(\xi^{1}, \xi^{2}\right)$ - the local interpolating (basis) functions; $\mathrm{N}$ is equal to $1,2, \ldots, \mathrm{N}_{(e)}\left(\mathrm{N}_{(e)}-\right.$ the number of the double points in the finite element); $\xi^{\alpha}-$ the arbitrary curvilinear coordinate system.

The covariant derivatives are approximated:

$$
\nabla_{\mathrm{k}} \delta U_{\bar{i}}=\frac{\partial \delta U_{-}^{-}}{\partial \xi^{\beta}}-\delta U_{k} \stackrel{(-)}{\Gamma_{\beta i}^{k}}=\left(\frac{\partial \Psi_{N}}{\partial \xi^{\beta}} \delta_{i}^{k}-\Psi_{N} \stackrel{(-)}{\Gamma_{\beta i}^{k}}\right) \delta U_{k}^{N} .
$$

The Variational equation for the finite element has the form as follows

$$
\begin{aligned}
& \int_{S_{(e)}}\left[\left(\delta t^{(-)} \bar{i}\left(\frac{\partial \Psi_{N}}{\partial \xi^{\beta}} \delta_{i}^{k}-\Psi_{N} \stackrel{(-)}{\Gamma_{\beta i}^{k}}\right)-\Psi_{N} \delta \vartheta^{-}\right) \delta \stackrel{(-)}{U} \stackrel{N}{k}^{k}\right. \\
& \left.+\left(\delta \stackrel{(+)}{t^{\beta} \bar{i}}\left(\frac{\partial \Psi_{N}}{\partial \xi^{\beta}} \delta_{i}^{k}-\Psi_{N} \stackrel{(-)}{\Gamma_{\beta i}^{k}}\right)-\Psi_{N} \delta \vartheta^{-\bar{k}}\right) \delta \stackrel{(+)}{U} \underset{k}{N}\right] d \stackrel{(-)}{S} \\
& -\int_{S_{(e)}}\left[\Psi_{N} \delta \kappa^{(-)}{ }^{-} \delta \stackrel{(-)}{U} \stackrel{N}{k}_{k}+\Psi_{N} \delta \kappa^{(+)} \stackrel{-(+)}{k} \underset{U}{U} \frac{N}{k}\right] d \stackrel{(-)}{S}
\end{aligned}
$$




$$
-\int_{\Gamma_{(e)}}\left[\Psi_{N} \delta \stackrel{(-)}{T}^{k} \delta \stackrel{(-)}{U} \underset{k}{\frac{N}{k}}+\Psi_{N} \delta \stackrel{(+)}{T}^{k} \delta \stackrel{(+)}{U} \underset{k}{\frac{N}{k}}\right] d \stackrel{(-)}{\Gamma}=0 .
$$

From which, after the substitution for the equation (10) and the finite elements coupling, we get the global equilibrium equations [6]:

$$
\begin{aligned}
& \sum_{(e)} \stackrel{(e)}{\Omega_{\Delta}^{N}}\left(\stackrel{(-)}{a} \underset{N M}{K L} \delta \stackrel{(-)}{M}_{L}^{M}+\stackrel{(-)}{a} \underset{N M}{K L} \delta \stackrel{(+)}{U}_{L}^{M}\right)=\sum_{(e)} \stackrel{(e)}{\Omega}{ }_{\Delta}^{N} \delta \stackrel{(-)}{P}{ }_{N}^{K}, \\
& \sum_{(e)} \Omega_{\Delta}^{(e)}{ }_{\Delta}\left(\stackrel{(+)}{a} \underset{N M}{K L} \delta \stackrel{(-)}{U} \underset{L}{M}+\stackrel{(++)}{a} \underset{N M}{K L} \delta \stackrel{(+)}{U} \frac{M}{L}\right)=\sum_{(e)}{ }^{(e)}{ }_{\Delta}^{N} \delta \stackrel{(+)}{P_{N}^{K}},
\end{aligned}
$$

The incidence relation $\Omega_{\Delta}^{N}$ accepts the value of 1 , if the local element node $\mathrm{N}$ coincides with the global node $(-)$

$\Delta$ of the related area $S$, and the value of 0 , in the ontrary case.

Solving the elastoplastic problems should be preceded by study of the temperature field of the shell. As already mentioned, the approximation of the threedimensional temperature field of the shell is also carried out through the temperature of the base surfaces:

$$
T\left(t, X^{1}, X^{2}, X^{3}\right)=\left(1-X^{3}\right) \stackrel{(-)}{T}\left(t, X^{1}, X^{2}\right)+X^{3} \stackrel{(+)}{T}\left(t, X^{1}, X^{2}\right) .
$$

Using the principle of Morse and Feshbach [7], we get the functional, by minimization of which, the temperatures of the base surfaces are determined. For the plates, the finite element formulation of the appropriate variational temperature problem results in a system of the ordinary differential equations:

$$
\begin{gathered}
2 b_{M N} \frac{\partial \stackrel{(-)}{T}^{M}}{\partial t}+b_{M N} \frac{\partial \stackrel{(+)}{T}{ }^{M}}{\partial t} \\
+\stackrel{(-)}{T}{ }^{M}\left(2 C_{M N}+d_{M N}+e_{M N}+2 f_{M N}\right) \\
+{ }^{(+)}{ }^{M}\left(C_{M N}-d_{M N}+f_{M N}\right)=P_{N}
\end{gathered}
$$

$$
\begin{gathered}
b_{M N} \frac{\partial \stackrel{(-)}{T}^{M}}{\partial t}+2 b_{M N} \frac{\partial \stackrel{(+)}{T}{ }^{M}}{\partial t} \\
+\stackrel{(-)}{T}{ }^{M}\left(C_{M N}-d_{M N}+f_{M N}\right)
\end{gathered}
$$

$$
+\stackrel{(+)}{T}^{M}\left(2 C_{M N}+d_{M N}+e_{M N}+2 f_{M N}\right)=S_{N}
$$

Where

$$
\begin{aligned}
& b_{M N}=\int_{(-)} c \rho \frac{H}{6} \Psi_{N} \Psi_{M} d S \text {, } \\
& C_{M N}=\int_{\substack{(-) \\
S}} \frac{H}{6}\left(\lambda_{X} \frac{\partial \Psi_{N}}{\partial X} \frac{\partial \Psi_{M}}{\partial X}+\lambda_{Y} \frac{\partial \Psi_{N}}{\partial Y} \frac{\partial \Psi_{M}}{\partial Y}\right) d S, \\
& d_{M N}=\int_{(-)}^{(-)} \lambda_{Z} H \Psi_{N} \Psi_{M} d S, e_{M N}=\int_{S_{1}} \alpha \Psi_{N} \Psi_{M} d S, \\
& f_{M N}=\int_{\substack{(-) \\
\Gamma_{1}}} \alpha \frac{H}{6} \Psi_{N} \Psi_{M} d \Gamma
\end{aligned}
$$

$$
\begin{aligned}
& P_{N}=\left(2 \stackrel{(-)}{Q}{ }^{M}+\stackrel{(+)}{Q}{ }^{M}\right) \int_{\substack{S \\
S}} \frac{h}{6} \Psi_{N} \Psi_{M} d S-\stackrel{(-)}{q} M \int_{\substack{(-) \\
S_{2}}} \Psi_{N} \Psi_{M} d S \\
& -\left(2 \stackrel{(-)}{q}{ }^{M}+\stackrel{(+)}{q} M\right) \int_{(-)} \frac{h}{6} \Psi_{N} \Psi_{M} d \Gamma+T_{H} \int_{\Gamma_{2}} \alpha \Psi_{N} \Psi_{M} d S \\
& +T_{H} \int_{(-)} \alpha \frac{H}{2} \Psi_{N} d \Gamma,
\end{aligned}
$$

$\lambda_{X}, \lambda_{Y}, \lambda_{Z}-$ the coefficients of thermal conductivity; $\mathrm{Q}-$ specific capacity (per unit volume) of the internal sources (sinks) of heat; c - specific thermal capacity; $\rho$-density; $(-)(+)$

$S_{1}, S_{1}$ - the parts of the boundary surfaces, on which the

$$
(-)(+)
$$

heat flows q are set; $S_{2}, S_{2}-$ the parts of the boundary surfaces, on which the heat-exchange is set (heatexchange coefficient $\alpha$; exterior temperature $T_{H}$ ).

When using the Crank-Nicolson finite-difference scheme [8], the system of differential equations (18) transfers into the system of linear algebraic equations:

$$
\begin{aligned}
& \stackrel{(e)}{\Omega_{\Delta}^{N}}\left[\stackrel{(-)}{T_{i}{ }^{M}}\left(\frac{2 b_{M N}}{\Delta t}+C_{M N}+\frac{d_{M N}+b_{M N}}{2}+f_{M N}\right)\right. \\
& \left.+\stackrel{(+)}{T}{ }_{i}^{M}\left(\frac{b_{M N}}{\Delta t}+\frac{C_{M N}-d_{M N}+f_{M N}}{2}\right)\right] \\
& =\stackrel{(e)}{\Omega_{\Delta}^{N}}\left[\stackrel{(-)}{T_{i-1}^{M}}\left(\frac{b_{M N}}{\Delta t}+\frac{C_{M N}-d_{M N}+f_{M N}}{2}\right)\right. \\
& \left.+\frac{1}{2}\left(P_{N i-1}+P_{N i}\right)\right] \\
& \stackrel{(e)}{\Omega_{\Delta}^{N}}\left[{ }_{T}^{(-)}{ }_{i}^{M}\left(\frac{b_{M N}}{\Delta t}+\frac{C_{M N}-d_{M N}+f_{M N}}{2}\right)\right. \\
& \left.+\stackrel{(+)}{T}_{i}^{M}\left(\frac{2 b_{M N}}{\Delta t}+C_{M N}+\frac{d_{M N}+e_{M N}}{2}+f_{M N}\right)\right] \\
& =\stackrel{(e)}{\Omega_{\Delta}^{N}}\left[\stackrel{(-)}{T_{i-1}^{M}}\left(\frac{b_{M N}}{\Delta t}-\frac{C_{M N}-d_{M N}+f_{M N}}{2}\right)\right. \\
& +\stackrel{(+)}{T}{ }_{i}^{M}\left(\frac{2 b_{M N}}{\Delta t}-C_{M N}-\frac{d_{M N}+e_{M N}}{2}+f_{M N}\right) \\
& \left.+\frac{1}{2}\left(S_{N i-1}+S_{N i}\right)\right]
\end{aligned}
$$

By this means, in order to find the node temperatures

${ }^{T}{ }_{i}{ }^{M}, \stackrel{(-)}{T}_{i}{ }^{M}$, at the end of the $\mathrm{i}$-th time step, it is necessary to solve the system of linear algebraic equations (19).

The developed mathematical model was used when studying the temperature and the strain-stress states of the plate during welding [9]. The plate consisting of steel $12 \times 18 \mathrm{H} 10 \mathrm{~T}$ with the size of $0,4 \times 0,4 \mathrm{~m}$ and the thickness of $0,01 \mathrm{~m}$, was smelting in the middle, by nonconsumable tungsten electrode. The arc speed $\mathrm{v}=0,0028 \mathrm{mps}$, heat effective capacity - $\mathrm{q}=3,670 \mathrm{~kW}$.

\section{Conclusions}


Using the theory of shells having several base surfaces, there has been obtained the system of differential equations describing the elastoplastic and variational temperature problems. In addition, using the method of finite elements, there has been developed the method for solving these problems. Also, the numerical study of the obtained mathematical model has been carried out.

\section{References}

1. Vinokurov V.A., Grigoryants A.G. Theory of welding deformations and stresses. M., MASHINOSTROENIE publishers, p. 235, 1984.

2. Makhnenko V.I. The computational methods of studying the kinetics of welding strains and stresses. Kiev, NAUKOVA DUMKA publishers, p. 320, 1976.

3. Tvalchrelidze A.K. The basic equations of the theory of shells with account for large deformations and shears // News of the Academy of Sciences of Georgia, No 1, pp. 53-56, 1986.
4. Birger I.A. Theory of plastic flow at non-isothermal loading // Mechanics and mechanical engineering, No 1, pp. 193-196, 1984.

5. Lurie A.I. The theory of elasticity. M. NAUKA publishers, p. 940, 1970.

6. Odon J. The finite elements in non-linear mechanics of continua. M., MIR publishers, p. 456, 1976.

7. Berdichevskiy V.P. The variational principles of continuum mechanics. M., MIR publishers, p. 447, 1983.

8. Gubeladze G.J., Kakabadze D.F., Tvalchrelidze A.K., Shubladze T.G. Numerical simulation of the nonstationary temperature fields in plates with the use of two base surfaces // Works of Georgian Technical University, Tbilisi, No 1(419), 1998.

9. Vinokurov V.A., Grigoryants A.G. Shubladze T.G. The experimental-calculating determination of internal strains and stresses during welding // Studying the properties and the elements of welded structures. M., Bauman Moscow Technical University, 1974. 